International Mathematical Forum, 2, 2007, no. 49, 2417 - 2436

\title{
A Geometric Property of the Octatonic Scale
}

\author{
Brian J. Mcartin \\ Applied Mathematics, Kettering University \\ 1700 West Third Avenue, Flint, MI 48504-4898, USA \\ bmccarti@kettering.edu
}

\begin{abstract}
An elementary geometric proof of Cohn's theorem on dyadic partitioning of musical scales is presented.
\end{abstract}

\section{Praeludium}

Most Western European music, past and present, is built upon the diatonic scale (witness Julie Andrews in The Sound of Music leading the von Trapp children: "Doe, a deer, a female deer..."). However, many alternative scales have found use throughout music history. For example [1], the Arabs in the 7th century A.D. utilized a scale known as "zer ef kend" ("string of pearls") composed of alternating half and whole steps (concepts which will be reviewed below). There was a resurgence of interest in this scale (now called the octatonic scale) during the late 19th and early 20th centuries led by Rimsky-Korsakov, Stravinsky, Debussy and Bartók.

Interest in the octatonic scale persists to this day. The influential 1974 album Red by King Crimson opens with composer/guitarist Robert Fripp burning through a sequence of ascending octatonic scales. In the 1997 movie adaptation of Robert A. Heinlein's science fiction classic Starship Troopers, Basil Poledouris' soundtrack employs pulsating brass and string octatonic scales to presage appearances of the "bugs".

This octatonic scale has the property that it may be partitioned into pairs of pitches of the same "type" in the maximum number of ways. This property (to be further elaborated upon below) is called dyadic partitioning and it allows the composer wide latitude in the construction of coherent musical structures. Cohn's Theorem [2] states that there is no other scale possessing this particular combinatorial property [3]. 
In this paper, the geometrical model that was presented in [4] will be employed in order to provide an elementary proof of this important musical theorem. However, in order to make the present account reasonably self-contained, we first review some requisite music theory fundamentals together with the basics of our geometric musical model.

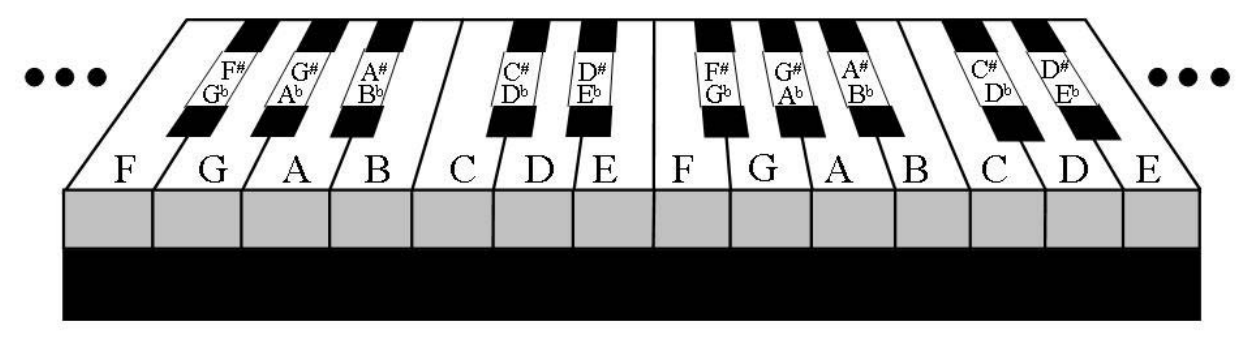

Figure 1: Piano Keyboard

\section{Musical Recapitulation}

Figure 1 displays a portion of the piano keyboard which will form the backdrop for our musical explorations. The piano is typically tuned in equal temperament, which means that any two adjacent keys (black or white) are at the same musical distance (interval) from one another. This common intervallic distance is referred to as a half-step (semitone) or minor second (m2). Thus, two adjacent white keys are a half-step apart while two white keys with an intervening black key are a whole step apart.

Table 1 contains the most common musical intervals. For example, from $C$ up (i.e. to the right) to $G$ is 7 half-steps and, thus, $C \uparrow G$ is a P5. Alternatively, from $C$ down (i.e. to the left) to $G$ is 5 half-steps so that $C \downarrow G$ is a P4. Consequently, we observe that P5 and P4 are complementary intervals in that they add up to an octave (P8). The self-complementary interval of a tritone $(\pi)$ will play a distinguished role in the ensuing exposition. 
Table 1: Musical Intervals

\begin{tabular}{|l|c||l|c|}
\hline Interval & No. of Half-steps & Interval & No. of Half-steps \\
\hline \hline unison (P1) & 0 & octave (P8) & 12 \\
\hline minor second (m2) & 1 & major seventh (M7) & 11 \\
\hline major second (M2) & 2 & minor seventh (m7) & 10 \\
\hline minor third (m3) & 3 & major sixth (M6) & 9 \\
\hline major third (M3) & 4 & minor sixth (m6) & 8 \\
\hline perfect fourth (P4) & 5 & perfect fifth (P5) & 7 \\
\hline tritone $(\pi)$ & 6 & tritone $(\pi)$ & 6 \\
\hline
\end{tabular}

A sharp $(\sharp)$ appearing after a note raises it by a half-step while a flat (b) lowers it by a half-step. In what follows, we invoke enharmonic equivalence by which we identify $C \sharp / D b$, etc. This might appear to be hair-splitting, yet if the piano were not tuned in equal temperament then these notes would be distinct. Moreover, we will assume octave equivalence, meaning that all $C$ 's are considered identical, and so on.

At first encounter, this last assumption seems puzzling. Indeed, if two $G$ 's are played on the piano, we perceive two distinct pitches ("a low $G$ " and "a high $G$ ") which "sound" very similar. In essence, we are saying that they sound enough alike to consider them as the same pitch-class ("a G"). The more mathematically seasoned reader will recognize the concept of equivalence class.

An immediate consequence of the assumption of octave equivalence is that any distinction between complementary intervals is nullified. Thus, for example, the $G$ above a given $C(\uparrow \mathrm{P} 5)$ and the $G$ below it $(\downarrow \mathrm{P} 4)$ are considered indistinguishable, so the corresponding intervals P4 and P5 must also be considered equivalent. Hence, only the left half of Table 1 need concern us in what follows.

The upshot of the above assumptions is that our musical universe, $\mathcal{U}$, is comprised of the 12 pitch-classes (pc's)

$\{C, C \sharp / D b, D, D \sharp / E b, E, F, F \sharp / G b, G, G \sharp / A b, A, A \sharp / B b, B\}$ (chromatic scale). We may number these "equally spaced" pc's and arrange them in a circle thereby arriving at the musical clock of Figure 2 together with its underlying modular arithmetic.

\section{Musical Geometry}

Any subset of our 12 pitch-class (pc) musical universe, $\mathcal{U}$ (a.k.a. the ag- 


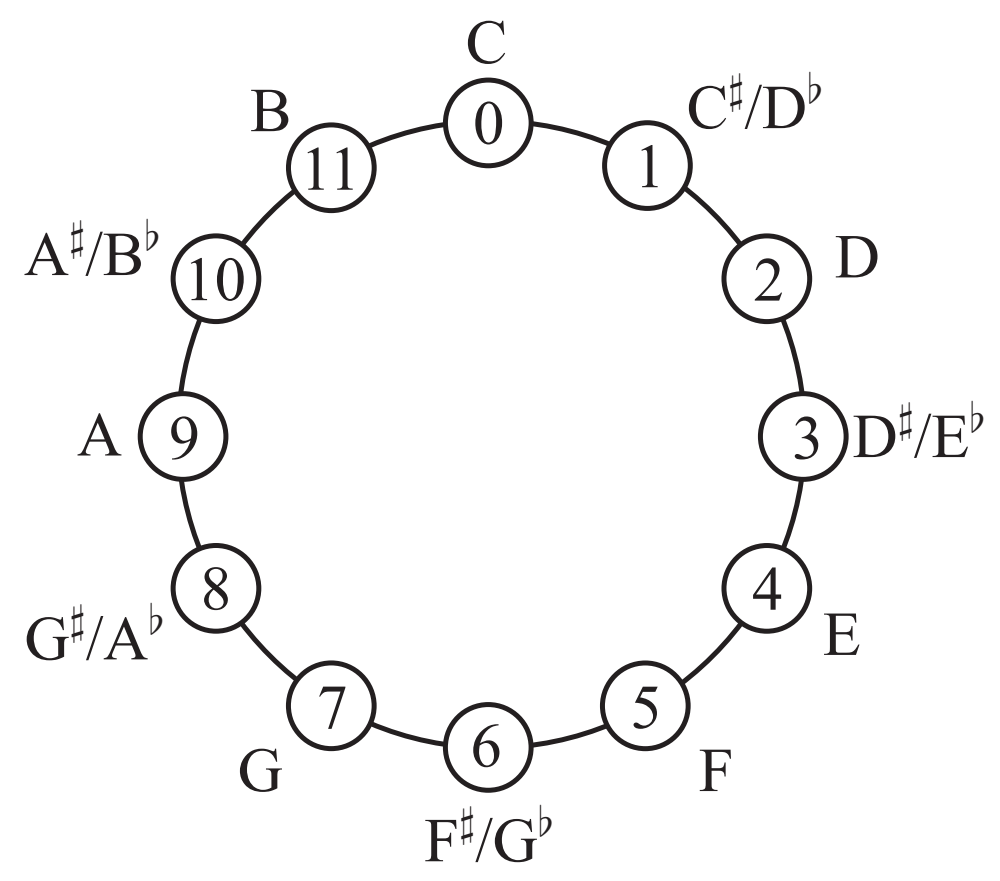

Figure 2: Musical Clock

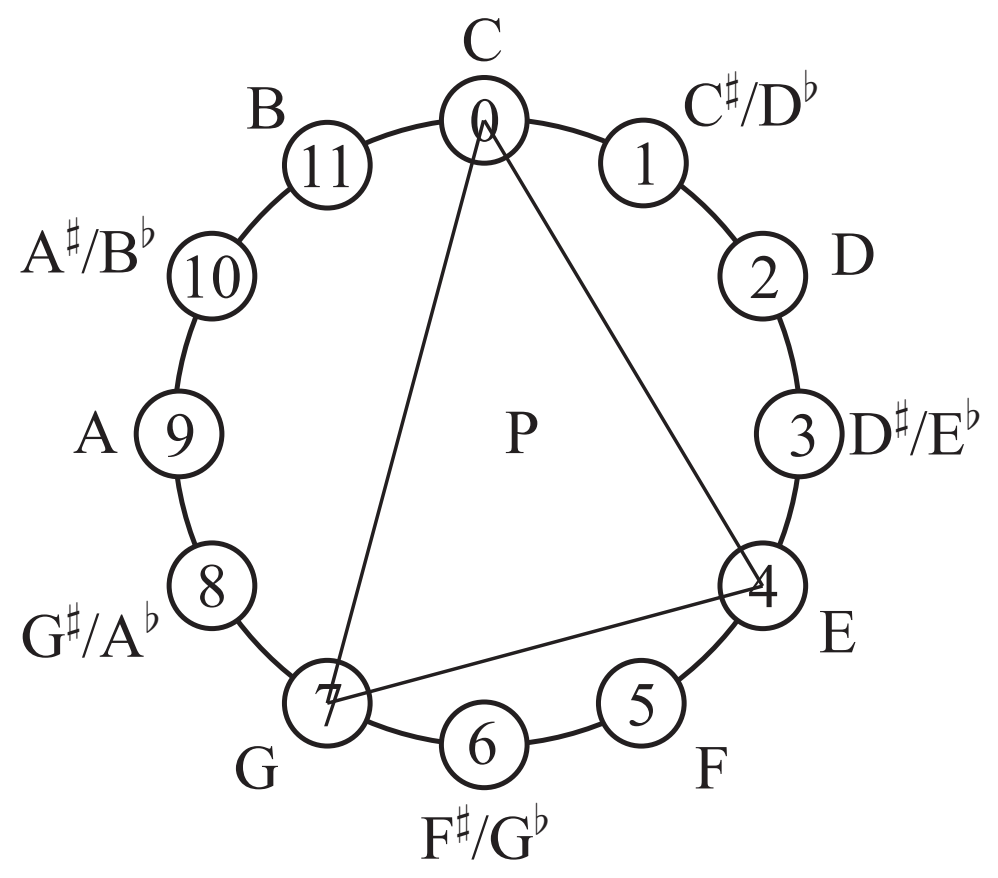

Figure 3: Pitch-Class Polygon 

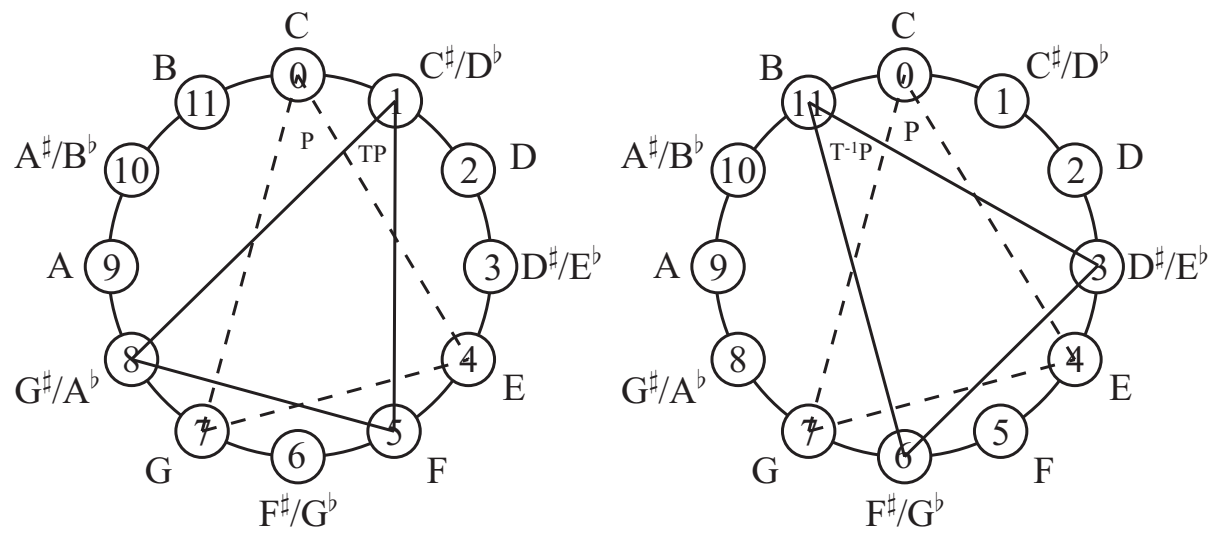

Figure 4: Pitch-Class Polygon Rotations
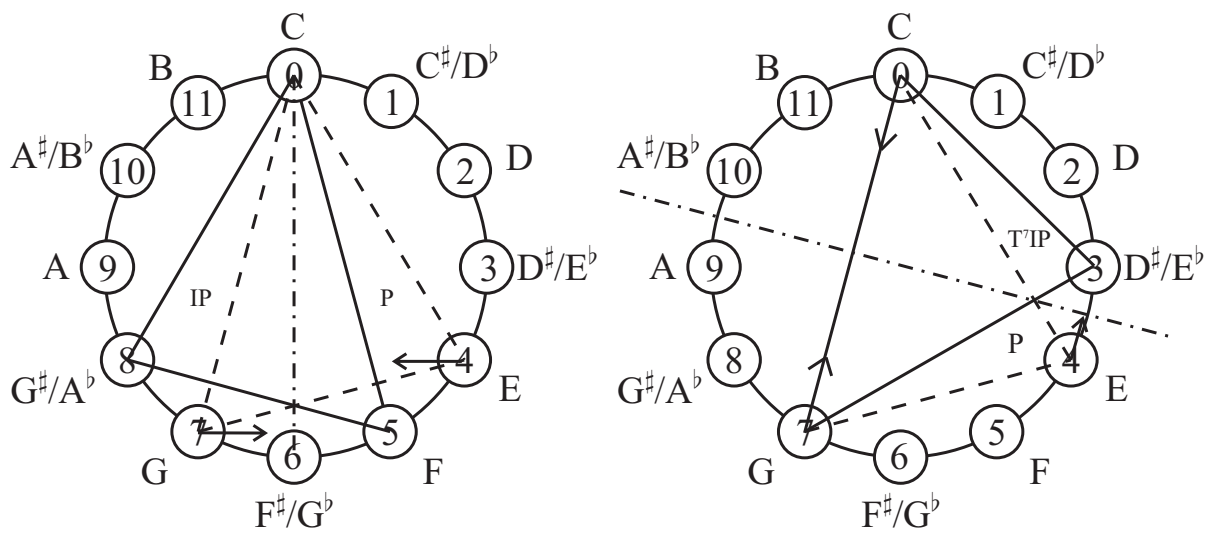

Figure 5: Pitch-Class Polygon Reflections 
gregate), may be identified with a (convex) polygon as follows: simply connect adjacent pc's of the subset (pc-set) by line segments on the musical clock. For example, consider the $C$ major triad, $\{C, E, G\}$, as represented in Figure 3. We may subject the pitch-class polygon, $P$, so obtained to rotations about an axis perpendicular to the clock and passing through its center. We will be exclusively concerned with rotations of an integer multiple of $30^{\circ}$ (i.e. $0^{\circ}, \pm 30^{\circ}, \pm 60^{\circ}, \ldots$ ), where the positive/negative direction is clockwise/counterclockwise, respectively.

Denote a clockwise rotation of $30^{\circ}$ by $T$ (Figure 4), so that $T P=\{C \sharp, F, G \sharp\}=$ $\{C \sharp, E \sharp, G \sharp\}$ (C\# major triad). We may represent all such rotations as $T^{n} \leftrightarrow$ $n \cdot 30^{\circ}$, where $n$ is an integer. Note that we may "multiply" (i.e. compose) these rotations using the laws of exponents: e.g. $T^{3}\left(T^{-2} P\right)=\left(T^{3} T^{-2}\right) P=T^{3-2} P=$ $T^{1} P=T P$. As such, these rotations of pc-polygons form an (abelian) group, the cyclic group of order $12, \mathcal{C}_{12}$ [5]. In musical parlance, such a rotation corresponds to a transposition.

Denote a reflection of a pc-polygon in the line passing through 0 - and 6 o'clock by $I$ (Figure 5$)$, so that $I P=\{F, A b, C\}$ ( $F$ minor triad). We may now concatenate reflections and rotations: e.g., $T^{7} I P=\{C, E b, G\}(C$ minor triad). Observe that this is equivalent to a pure reflection in a line which has been rotated by $105^{\circ}$. Mathematically, these combinations of reflections and rotations of pc-polygons comprise $\mathcal{D}_{12}$, the twelfth dihedral group (of order 24) [5]. Musically, reflections correspond to inversions, although the reader is cautioned that this term possesses multiple musical connotations [6].

\section{Octatonic Scale}

Figure 6 displays the septachord known as the $a$ minor scale, $\mathcal{N}:=$ $\{A, B, C, D, E, F, G\}$, composed of the collection of white piano keys together with the corresponding solfeggio syllables $l a, t i$, do, re, mi, fa, so. Any of its twelve transpositions or twelve inversions is referred to as a natural minor scale [6]. Because it possesses one reflectional symmetry (about the dashed axis) and one rotational symmetry (the identity transformation), it follows from the Orbit-Stabilizer Theorem [7, p. 140] that there are $\frac{24}{2}=12$ distinct (though not disjoint) such scales.

If we raise $f a$ and $s o$ by a half-step to $f i$ and si, respectively, then we obtain the variant pitch-class collection known as the ascending melodic minor scale [6], $\mathcal{M}$, of Figure 7 . Observe the consequent reflection of the axis of symmetry about the vertical axis passing through the center of the musical clock. If we now allow $m i$ to bifurcate by simultaneously lowering and raising it by a half-step then the result is the octachord called the octatonic scale, $\mathcal{O}$, of Figure 8. 


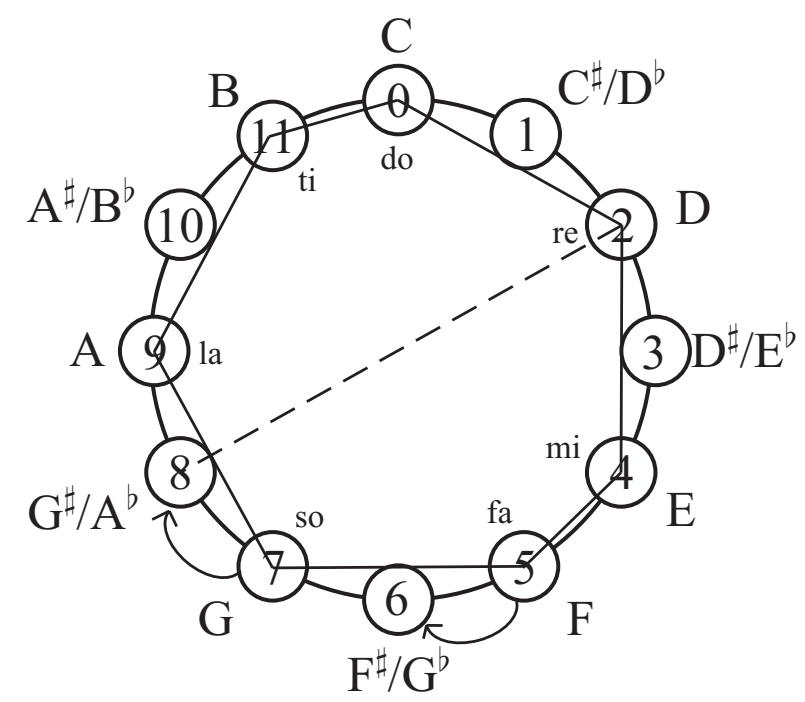

Figure 6: Natural Minor Scale

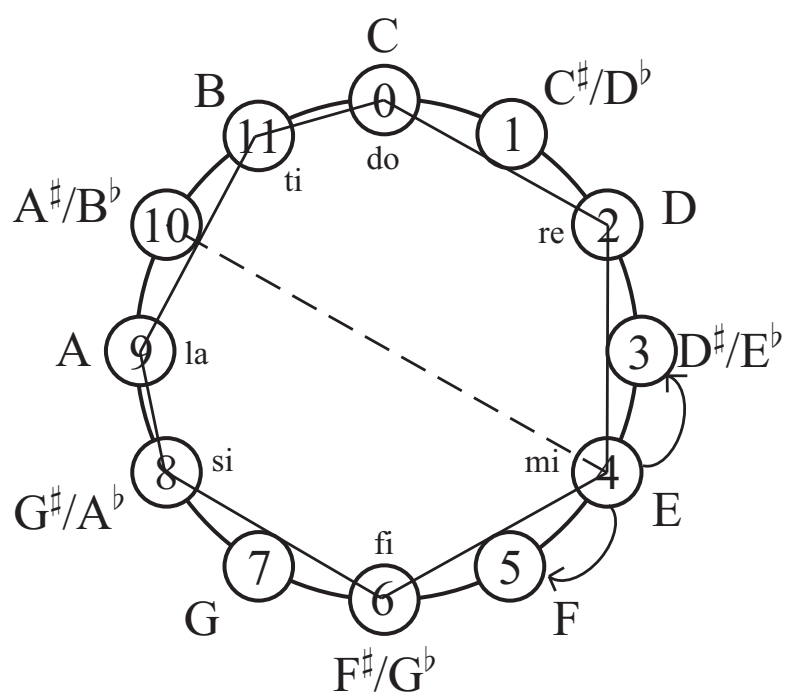

Figure 7: Ascending Melodic Minor Scale 


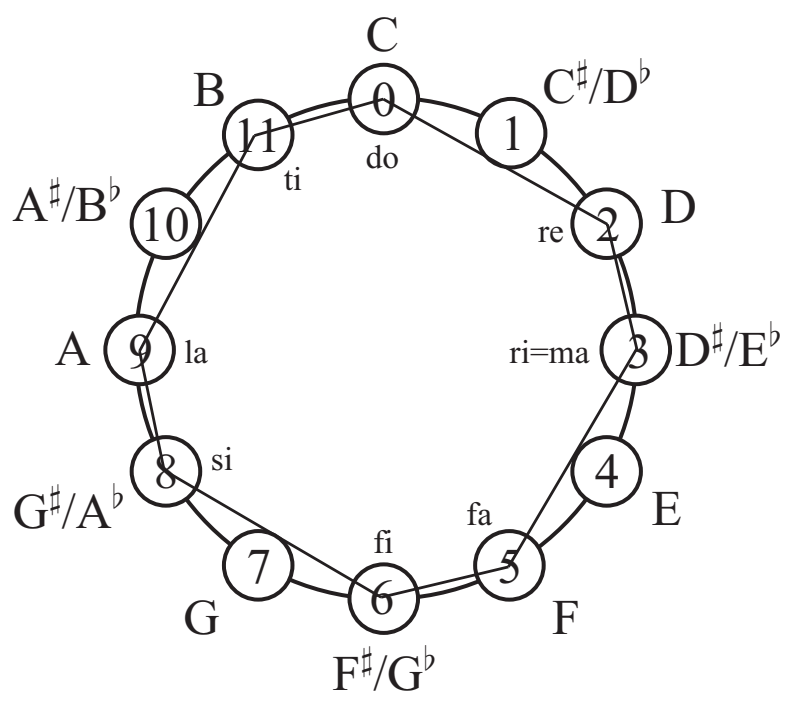

Figure 8: Octatonic Scale

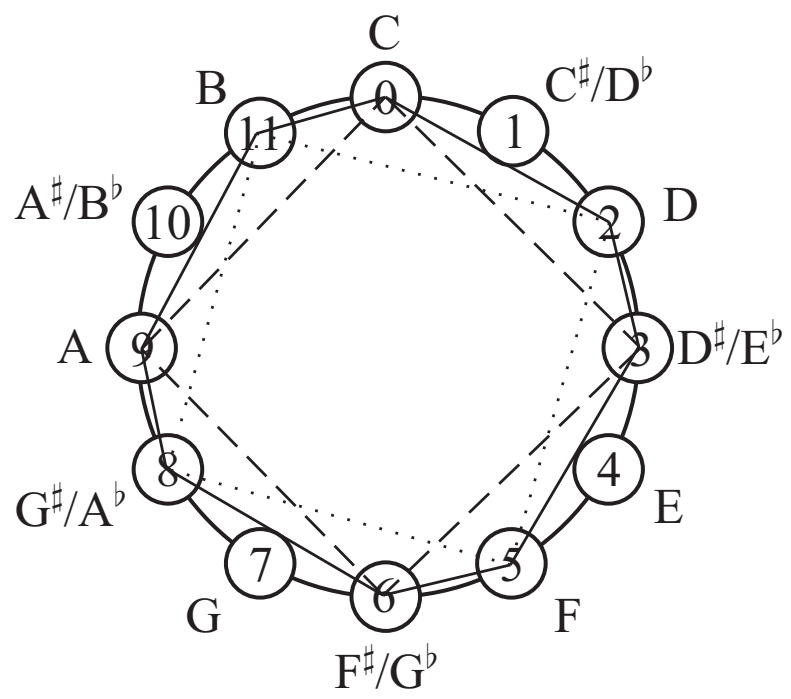

Figure 9: Diminished Scale 


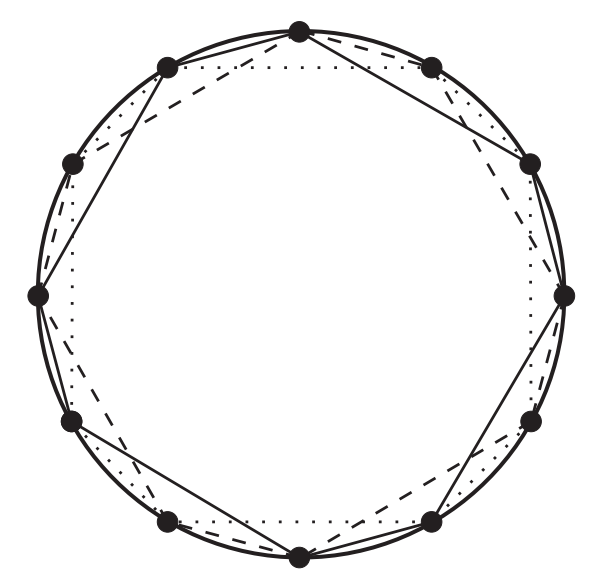

Figure 10: Three Octatonic Scales

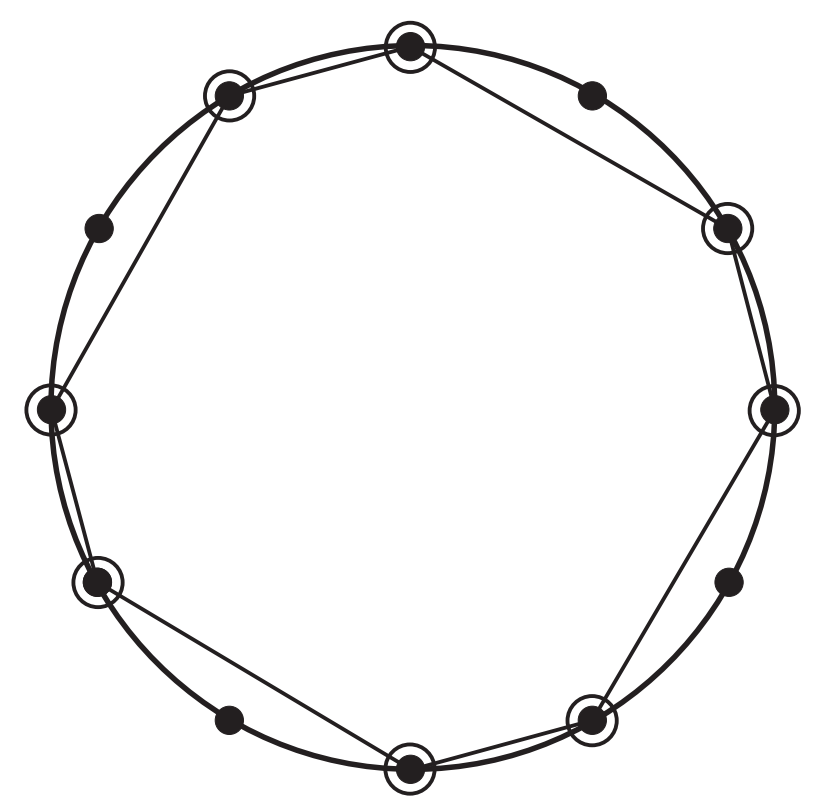

Figure 11: Prototype Octatonic Scale 
Note that, as is shown in Figure 9, the octatonic scale may be partitioned into two squares (fully diminished seventh chords [6]). For this reason, jazz musicians have dubbed this the diminished scale. Because this pitchclass polygon possesses four rotational and four reflectional symmetries, the Orbit-Stabilizer Theorem implies that there will be $\frac{24}{8}=3$ such octatonic scales as displayed in Figure 10. We will confine our attention to the prototypical octatonic scale of Figure 11 as the other two may be obtained from it by clockwise/counterclockwise rotation by a half-step.

\section{$5 \quad$ Dyadic Partitioning}

As Figure 11 reveals, the octatonic scale is highly symmetric. In fact, its symmetry group coincides with that of the square (the octic group [8]). The importance of such symmetry in music is widely recognized $[1,9]$. The remainder of this paper will explore a subtle consequence of this underlying symmetry of the octatonic scale [2]. In this and subsequent figures, numerical and literal labelling of pitch-classes have been suppressed as they are superfluous to the ensuing geometrical development.

But first, we require some definitions. Any pair of pitch-classes will be referred to as a dyad. Such dyads will be characterized by the interval formed by their component pitch-classes. Thus, given our prior assumption of octave equivalence, there are only six possible dyad classes which are shown in Figure 12. Any pitch-class collection is said to be dyadically partitioned by one of these six dyad classes if it may be written as the disjoint union of dyads of the same class.

For example, Figure 13 portrays the dyadic partitioning of the octatonic scale of Figure 11 by minor seconds. There, the members of the octatonic scale have been circled and the minor seconds of the partition represented by line segments. This convention is maintained in Figure 14, which displays the corresponding dyadic partition of the octatonic scale by major seconds.

Whereas these two dyadic partitions are unique, Figure 15 shows that the octatonic scale may be dyadically partitioned by minor thirds in four distinct ways. However, turning to Figures 16, 17 and 18, we see that the octatonic scale may be dyadically partitioned by major thirds, perfect fourths and tritones in only one way, respectively. 


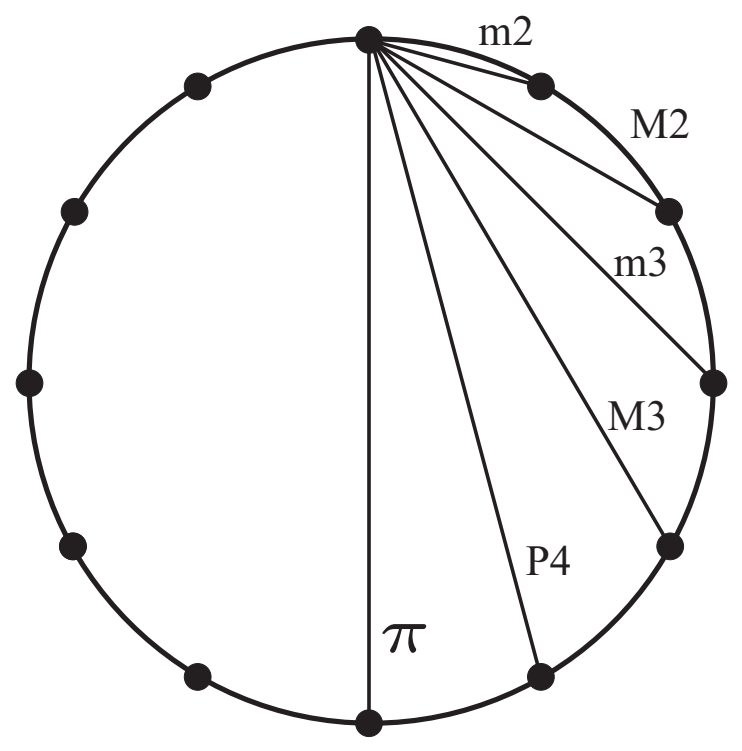

Figure 12: Six Dyad Classes

Figures 13-18 taken in toto reveal that the octatonic scale may be dyadically partitioned by each of the six available dyad classes. This raises the interesting question: Are there any other pitch-class collections possessing this same property?

\section{Cohn's Theorem}

A negative response to this question has been provided by Richard Cohn $[2]$.

Theorem 1 (Cohn's Theorem on Dyadic Partitioning) The only pitchclass collection in the twelve-tone equally tempered musical universe that can be dyadically partitioned into all six dyad classes is the Octatonic Collection.

Before embarking on a proof of this important result, a matter of mathematical aesthetics needs to be addressed. Up to rotation and reflection, there are precisely 223 different pitch-class collections and detailed descriptions of each of them have been tabulated [10]. A brute force approach to proving musical results such as Cohn's Theorem entails exhaustive inspection of all 223 pc's to see if they possess the required property. However, such a ham-fisted attack would hardly qualify for Erdös "Book" of most beautiful proofs [11]. Indeed, such aesthetic considerations are at the core of many mathematicians' discomfort with the proof by Haken and Appel of the Four Color Theorem [12]. Consequently, we shall strive for a measure of elegance in our demonstration. 


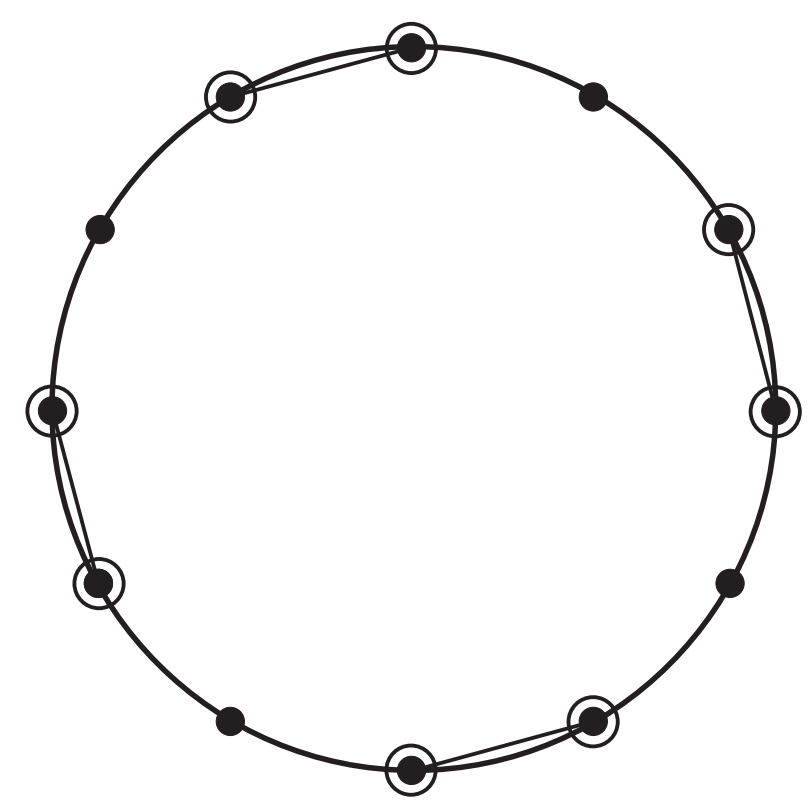

Figure 13: Dyadic Partitioning of Octatonic Collection: $\mathrm{m} 2$

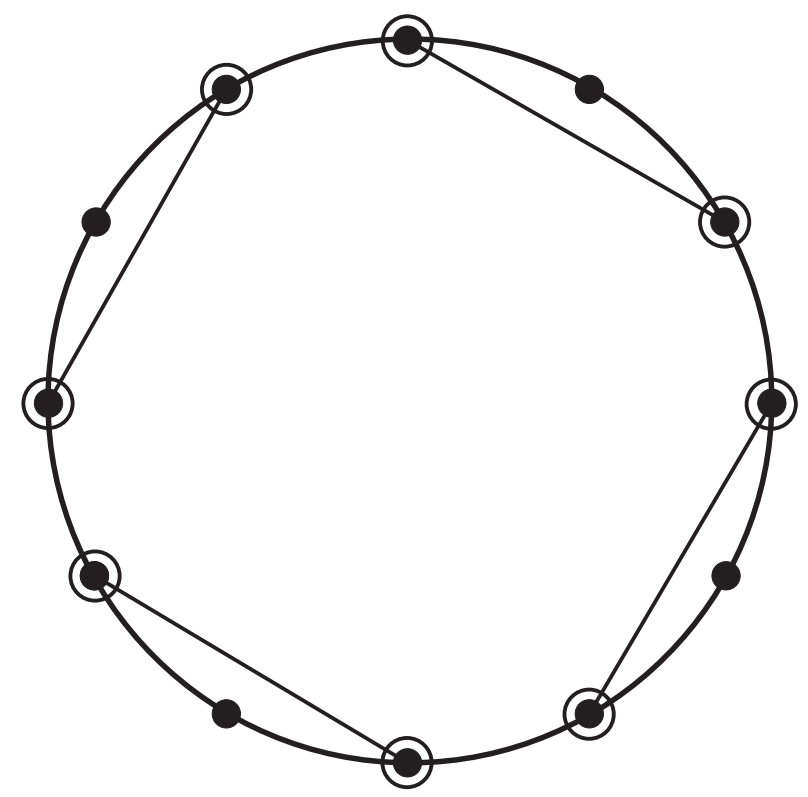

Figure 14: Dyadic Partitioning of Octatonic Collection: M2 

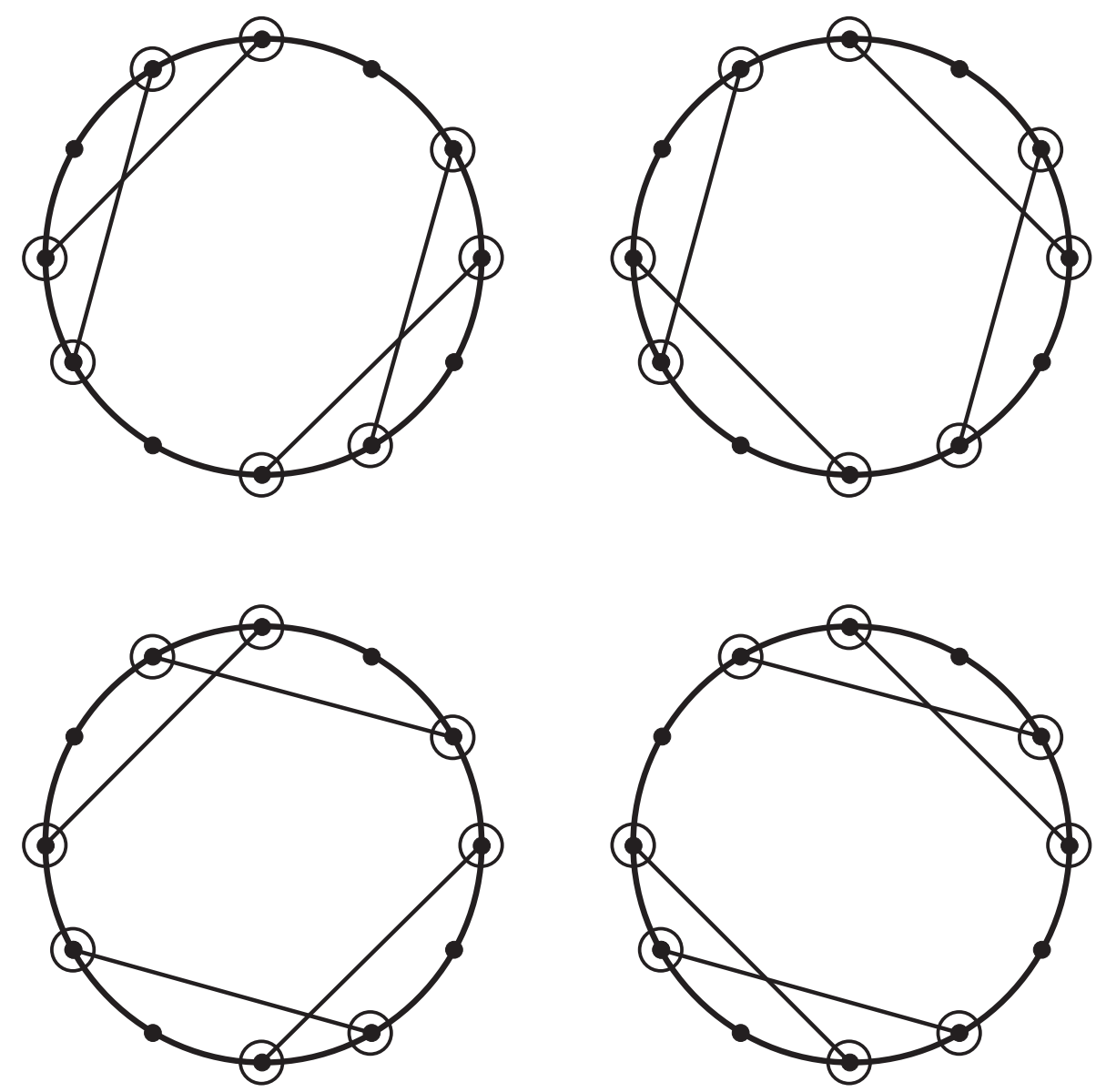

Figure 15: Dyadic Partitioning of Octatonic Collection: m3 


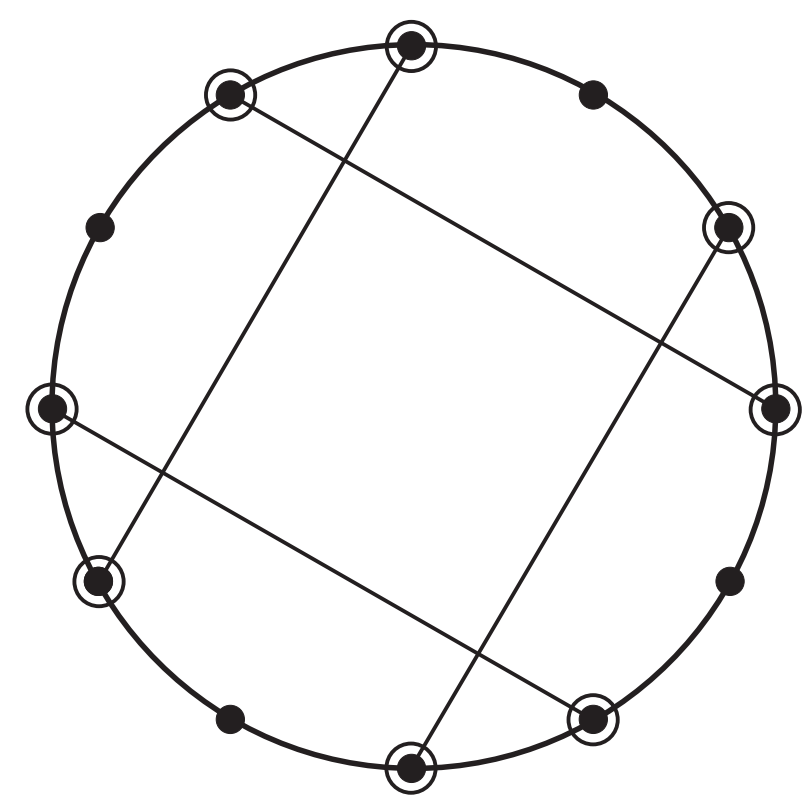

Figure 16: Dyadic Partitioning of Octatonic Scale: M3

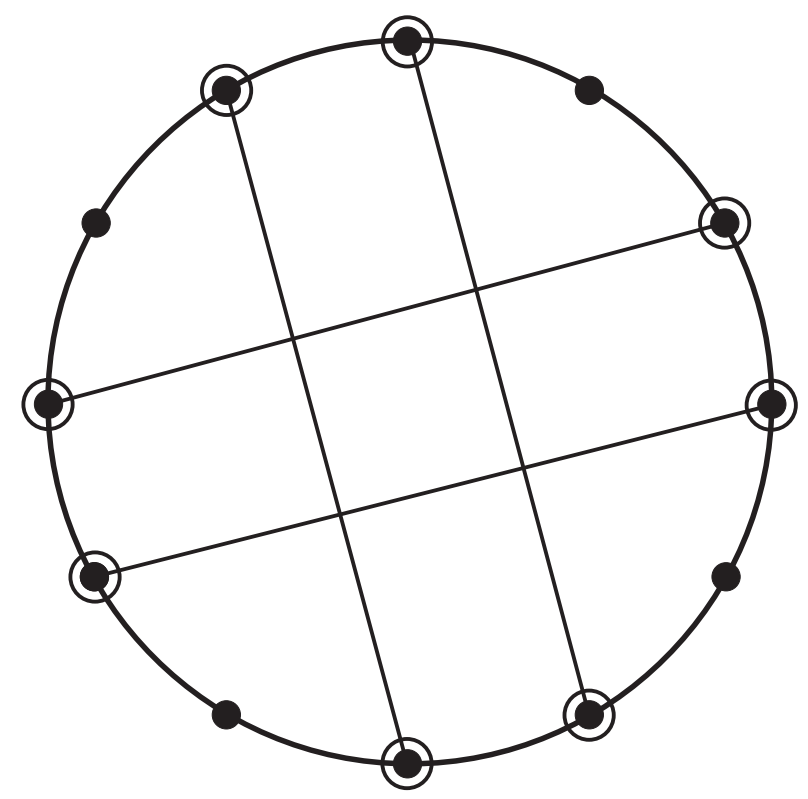

Figure 17: Dyadic Partitioning of Octatonic Collection: P4 
Proof: First of all, since we desire partitions by dyads, it is clear that $n$, the cardinality of the pitch-class collection, must be even. Moreover, if $n=2$, 4 or 6 then there simply are not sufficiently many dyads. For example, in the dyadic partitioning of a hexachord, we require at least $3 \times 6=18$ dyads yet a hexachord contains only $\left(\begin{array}{l}6 \\ 2\end{array}\right)=15<18$ dyads.

We defer consideration of octachords and instead move on to decachords. If a decachord is to be dyadically partitioned by tritones then it must contain five of the six possible tritones which implies that the two omitted pitchclasses must lie at the extremities of the "missing" tritone and, hence, must be diametrically opposed as in Figure 19. But, the decachord shown there has no dyadic partitioning by minor seconds. This follows because, for example, the required minor seconds connecting 1-2 o'clock and 4-5 o'clock preclude the presence of the two minor seconds containing 3 o'clock since the elements of the partition must by definition be disjoint.

Turning now to dyadic partitioning of the dodecachord, Figure 20 highlights the fact that the inclusion of any particular major third (shown solid) excludes the other two major thirds (shown dashed) emanating from its extremities. These two major thirds meet at a common pitch-class which is thereby excluded from the partition.

Consequently, our focus has been narrowed to octachords which, of course, must omit four pitch-classes such as those shown circled in Figure 21. The remaining eight pitch-classes must contain four of the six possible tritones and hence two tritones must be excluded which implies that the omitted pitches must be comprised of two antipodal pairs. Thus, the configuration shown in Figure 21 cannot be dyadically partitioned by tritones. Therefore, modulo rotations, the only configurations that can be so partitioned either appear in Figure 22 or are reflections thereof about a vertical axis passing through the center of the musical clock.

The left-most configuration of Figure 22 cannot be dyadically partitioned by minor thirds since, for example, the two minor thirds containing 3 o'clock both contain an excluded pitch-class. Likewise, the middle configuration of Figure 22 cannot be dyadically partitioned by minor seconds since, for example, the two minor seconds containing 1 o'clock both contain an excluded pitchclass. Finally, this brings us to the right-most configuration of Figure 22 which is none other than the octatonic scale! Q.E.D.

\section{Coda}

Upon reflection, the preceding development is really quite remarkable. With only the simplest of mathematical tools, we have established an important musical property possessed by one and only one collection of musical 


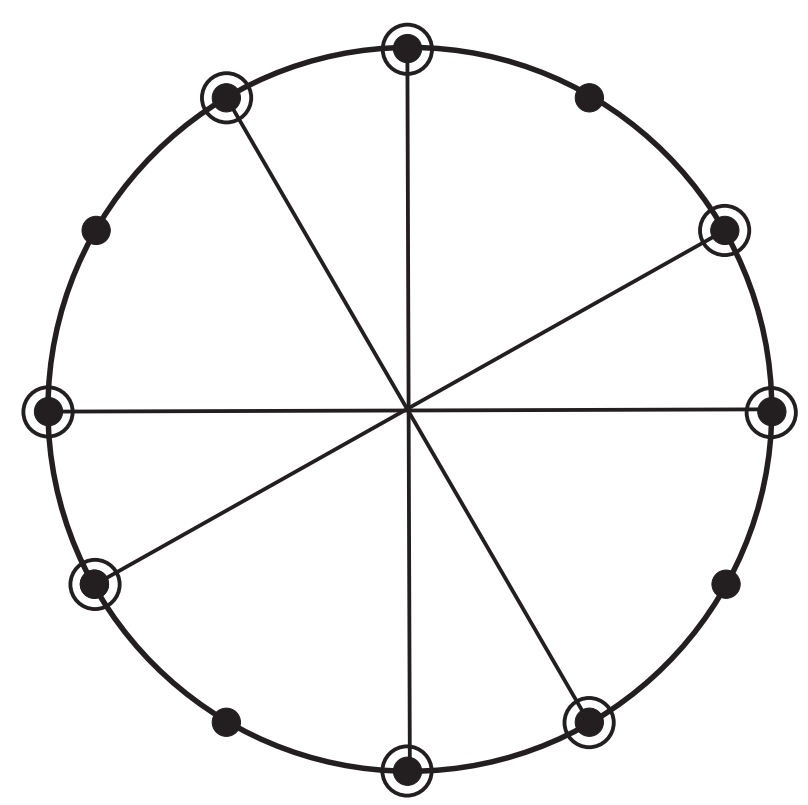

Figure 18: Dyadic Partitioning of Octatonic Collection: $\pi$

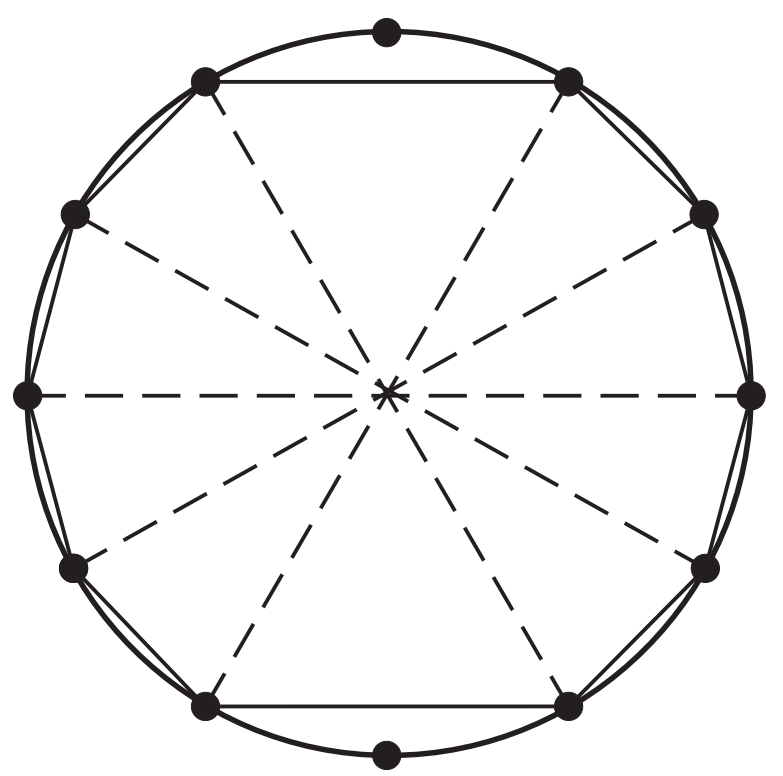

Figure 19: Dyadic Partitioning of Decachord: $\sim m 2$ 


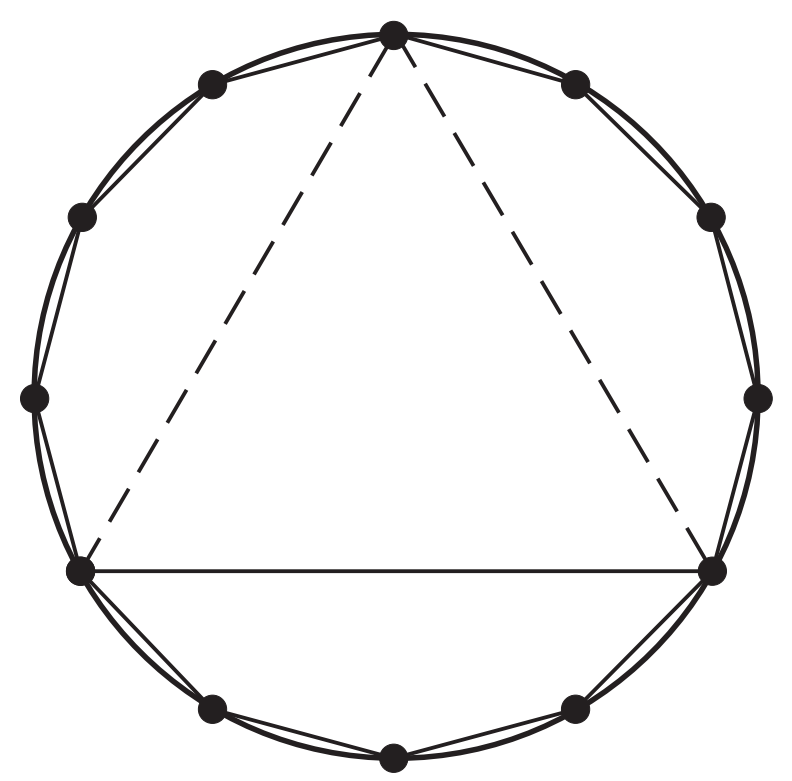

Figure 20: Dyadic Partitioning of Aggregate: M3

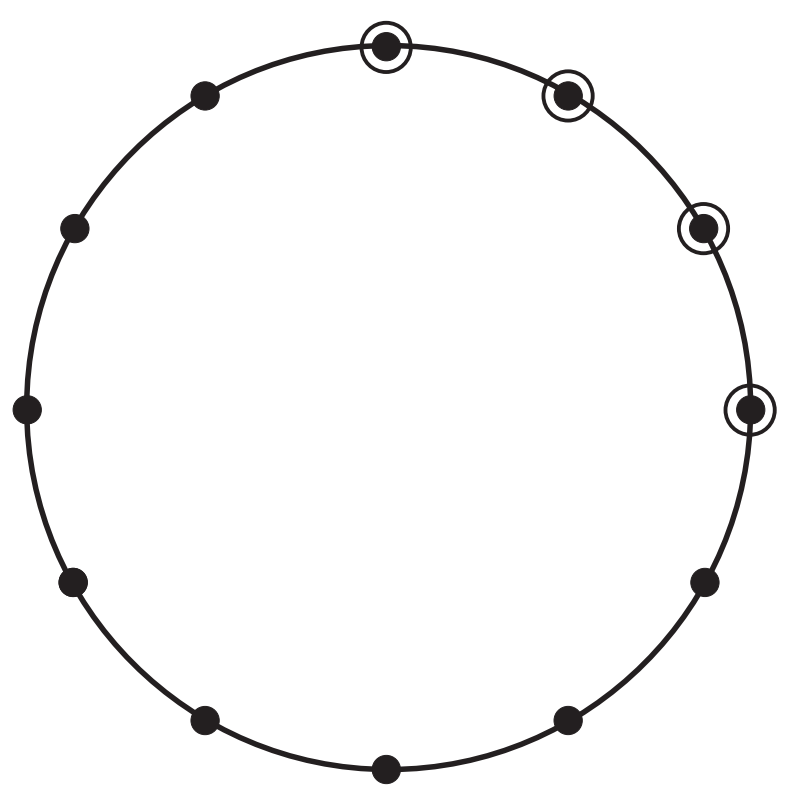

Figure 21: Dyadic Partitioning of Octachords - I 
pitches - the octatonic scale! Pondering the complete graph of eight vertices [13], $\mathcal{K}_{8}$, in Figure 23, one can only imagine what other precious jewels lie hidden within the intricate tapestry of the octatonic scale.

For the reader interested in further mathemusical exploration, [4] and [14] are highly recommended. For a different flavor of geometrical investigation of musical structure, [15] and [16] would handsomely repay careful study.

\section{Acknowledgement}

The author expresses heartfelt thanks to Mrs. Barbara McCartin for her invaluable assistance in the production of this paper. 


\section{References}

[1] Joseph Schillinger, The Mathematical Basis of the Arts, Da Capo, 1976.

[2] Richard Cohn, "Bartók's Octatonic Strategies: A Motivic Approach", Journal of the American Musicological Society, Vol. XLIV, No. 2, Summer 1991, pp. 262-300.

[3] Michael Keith, From Polychords to Polya: Adventures in Musical Combinatorics, Vinculum, 1991.

[4] Brian J. McCartin, "Prelude to Musical Geometry", College Mathematics Journal, Vol. 29, No. 5, November 1998, pp. 354-370.

[5] Israel Grossman and Wilhelm Magnus, Groups and Their Graphs, MAA, 1964.

[6] Patrick Miller, Study Guide for Tonal Harmony, Hartt School of Music, Sept. 1990.

[7] Joseph A. Gallian, Contemporary Abstract Algebra, Third Edition, D. C. Heath and Co., 1994.

[8] John B. Fraleigh, A First Course in Abstract Algebra, Addison-Wesley, 1972.

[9] H. S. M. Coxeter, "Music and Mathematics", The Mathematics Teacher, March 1968, pp. 312-320.

[10] John Rahn, Basic Atonal Theory, Schirmer, 1980.

[11] Paul Hoffman, The Man Who Loved Only Numbers, Hyperion, 1998.

[12] Robin Wilson, Four Colors Suffice, Princeton, 2002.

[13] Oystein Ore, Graphs and Their Uses, MAA, 1990.

[14] Michael J. Schiano, "The Role of Musical Set Theory in Undergraduate Education", College Music Symposium, Vol. 39, Fall 1999, pp. 64-86.

[15] Wilfrid Hodges, "The Geometry of Music", Music and Mathematics: From Pythagoras to Fractals, J. Fauvel, R. Flood, and R. Wilson (Editors), pp. 92-111, Oxford, 2003.

[16] Guerino Mazzola, "The Topos Geometry of Musical Logic" in Mathematics and Music: A, Diderot Mathematical Forum, G. Assayag, H. G. Feichtinger, and J. F. Rodrigues (Editors), pp. 199-213, Springer, 2002.

Received: March 4, 2007 

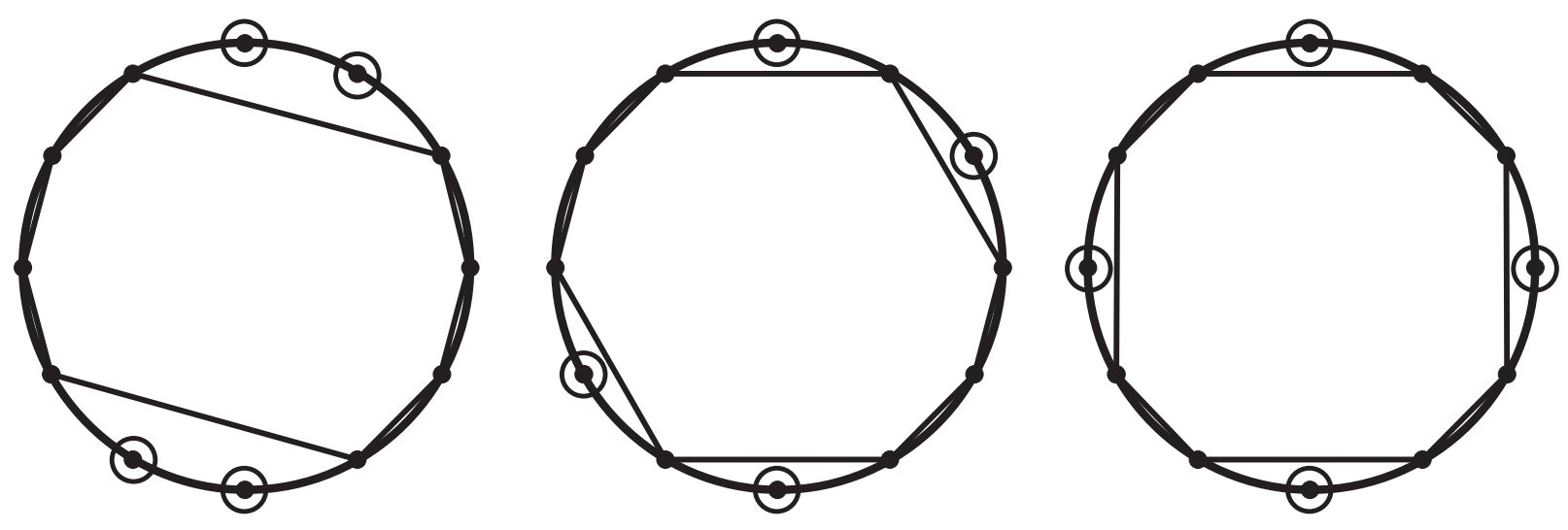

Figure 22: Dyadic Partitioning of Octachords - II

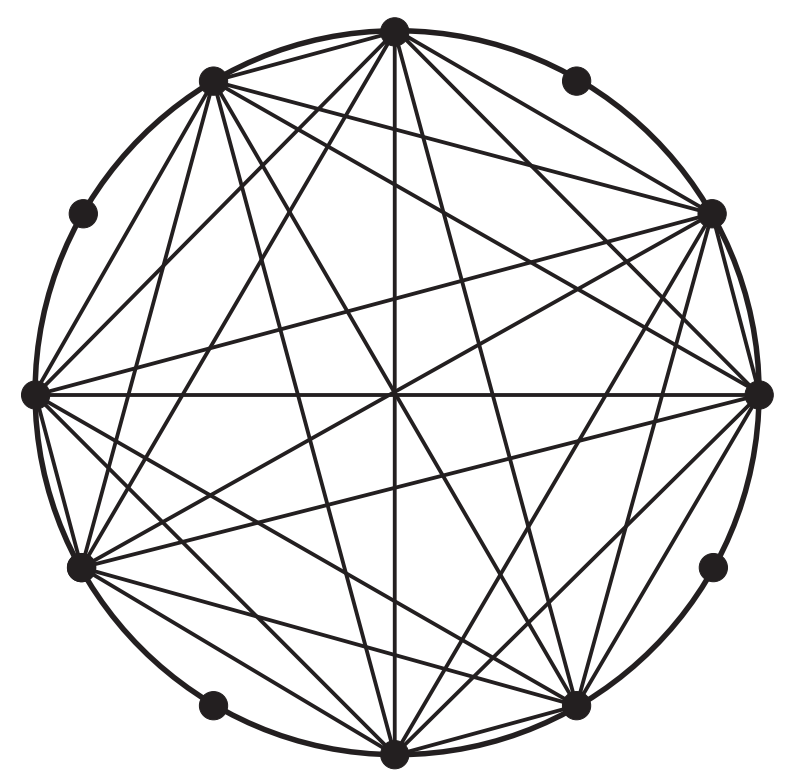

Figure 23: Geometric Structure of the Octatonic Scale 